TRANSACTIONS OF THE

AMERICAN MATHEMATICAL SOCIETY

Volume 352, Number 4 , Pages 1635-1645

S 0002-9947(99)02372-7

Article electronically published on May 3, 1999

\title{
A THEOREM ON SMOOTHNESS- BASS-QUILLEN, CHOW GROUPS AND INTERSECTION MULTIPLICITY OF SERRE
}

\author{
S. P. DUTTA
}

\begin{abstract}
We describe here an inherent connection of smoothness among the Bass-Quillen conjecture, the Chow-group problem and Serre's Theorem on Intersection Multiplicity. Extension of a theorem of Lindel on smoothness plays a key role in our proof of the Serre-multiplicity theorem in the geometric (resp. unramified) case. We reduce the complete case of the theorem to the above case by using Artin's Approximation. We do not need the concept of "complete Tor". Similar proofs are sketched for Quillen's theorem on Chow groups and its extension due to Gillet and Levine.
\end{abstract}

In order to prove the geometric case of the Bass-Quillen conjecture for projective modules over polynomial rings, Lindel [Li] came up with an elegant characterization of a special case of smoothness for regular local rings obtained by localizing a finitely generated $k$-algebra ( $k$ a field) at a maximal ideal. Afterwards Nashier Nas extended Lindel's idea to prove a structure theorem for smoothness (special type) with some descent data for any regular local ring essentially of finite type over a field $k$. In [D2], we pointed out that Nashier's theorem can be easily generalized to regular local rings $A$ essentially of finite type and smooth over a discrete valuation ring (henceforth d.v.r.) $V$ such that $A / m_{A}$ is separately generated over $V / m_{V}$. Using these generalizations along with Claborn and Fossum's $\mathrm{C}-\mathrm{F}$ work on Chow groups, we produced [D2] new proofs of Quillen's theorem on Chow groups for regular local rings essentially of finite type over a field and its extension due to Gillet and Levine for above rings smooth over a d.v.r. $V$. Our approach was totally different from that of Quillen $[\mathrm{Q}]$. In the d.v.r. case our proof was a bit restricted-we needed $A / m_{A}$ to be separably generated over $V / m_{V}$.

In this note we are going to produce a new proof of Serre's Theorem on Intersection Multiplicity in which the above generalizations of Lindel's result play a very significant role. We know, by Serre's work, that the study of intersection multiplicity in an affine space over a field or a d.v.r. reduces to the study of $\chi(A / P, A / \mathfrak{q})$, where $A$ is a regular local ring essentially of finite type over a field $k$ or a d.v.r. $V$ and $P, \mathfrak{q}$ are prime ideals of $A$ such that $\ell(A / P+\mathfrak{q})<\infty$. In order to prove both the equicharacteristic case and the unramified case, Serre reduced the problem to a similar problem on a power series ring; he came up with the notion of "complete Tor", and he used it along with the method of diagonalization to finish his proof. But our attack comes from a completely different viewpoint. We reduce

Received by the editors September 9, 1997.

1991 Mathematics Subject Classification. Primary 13D02; Secondary 13 H10.

This research was partially supported by an N.S.A. grant and an N.S.F. grant. 
the whole problem, both in the equicharacteristic case and in the unramified case, to a similar problem over a regular local ring obtained by localizing a polynomial ring at the most obvious maximal ideal and use the method of diagonalization to solve it. For the solution of Serre-multiplicity on power series rings over $k$ or $V$, we reduce the problem to arbitrary regular local rings essentially of finite type and smooth over $k$ or $V$ and then finish the proof by the technique mentioned above. For this reduction, Artin's approximation plays a key role. After this manuscript was prepared we got a preprint by Berthelot $[\mathrm{B}]$ in which he proved non-negativity of Serre-multiplicity following an exposition of Gabber's work. In his reduction of the complete case to the essentially finite case, Berthelot used spectral sequences which we do not need at all in our work. Hochster has recently shown that our technique can produce a direct reduction of the complete ramified case to the essentially finite type ramified case $[\mathrm{H} 2$. Note that Serre's theorem over arbitrary regular local rings follows directly from the complete case. We are really surprised to find that a structure theorem on a special case of smoothness can be such a major factor for three outstanding problems.

In Section 1, we state Lindel's result, Nashier's extension and our generalization. For the sake of completeness of this paper we provide a proof of this generalization. In Section 2, we furnish our proof of Serre's theorem in the geometric case. In Section 3, we do the same for the complete case. For the convenience of the reader we give a brief sketch of our proof of Quillen's theorem on Chow groups and its extension due to Gillet-Levine in Section 4.

A final remark. The approach, we emphasize here, also leads to a new proof of the following result due to Serre: Let $A$ be a regular local ring, and let $M, N$ be two finitely generated modules such that $\ell\left(M \otimes_{A} N\right)<\infty$. Then $\operatorname{dim} M+\operatorname{dim} N \leq$ $\operatorname{dim} A$.

First we state Lindel's result

1.1 Theorem ([Li, Proposition 2]). Let $A$ be an affine domain over a field $k$ with $d=\operatorname{dim} A \geq 1$. Let $m$ be a maximal ideal of $A$ such that $A_{m}$ is a regular local ring and the residue class field $K=A / m$ is a separable algebraic extension of $k$. Then there exist elements $x_{1}, \ldots, x_{t}$ in $A$ such that the following hold:

(i) $A=k\left[x_{1}, \ldots, x_{t}\right], m=\left(f\left(x_{1}\right), x_{2}, \ldots, x_{t}\right)$ with $f\left(x_{1}\right) \notin m^{2}$, where $f$ is the minimal polynomial of the image of $x_{1}$ in $K$,

(ii) $A_{m}$ is an étale neighborhood of $B_{n}$ where $B=k\left[x_{1}, \ldots, x_{d}\right]$ and $n=m \cap B$.

Next we state Nashier's extension of the above result [Nas]. It plays a crucial role in our proofs in Section 2 and Section 3.

1.2 Theorem $([\mathrm{N}])$. Let $(A, m, K)$ be a regular local ring essentially of finite type over a field $k$ such that $K / k$ is a finite separable extension. Let $a(\neq 0) \in m^{2}$. Then there exists a regular local ring $(B, n, K) \subset(A, m, K)$ with the following properties:

(1) $B$ is a localization of a polynomial ring $k\left[X_{1}, \ldots, X_{d}\right]$ at a maximal ideal $n$ of the type $\left(f\left(X_{1}\right), X_{2}, \ldots, X_{d}\right)$, where $f$ is a monic irreducible polynomial in $k\left[X_{1}\right]$. Moreover $B \rightarrow A$ is étale.

(2) There exists an element $h$ in $B \cap a A$ such that $B /(h B) \rightarrow A /(a A)$ is an isomorphism. 
For a proof we refer the reader to $\mathrm{Nas}$. We comment that by a standard technique (see proof of Theorem 1.3) one can recover the case when $K$ is separably generated over $k$.

Now we state our generalization and prove it. It is an extension of Nashier's theorem to the d.v.r. case.

1.3 Theorem ([D2]). Let $(A, m, K)$ be a regular local ring of dimension $d+1$, essentially of finite type and smooth over an excellent d.v.r. $(V, \pi)$ such that $A / m$ is separately generated over $V / \pi V$. Let $a(\neq 0) \in m^{2}$ be such that $a \notin \pi A$ (i.e., $\{\pi, a\}$ form an $A$-sequence). Then there exists a regular local ring $(B, n, K) \subset(A, m, K)$ such that

(1) $B$ is a localization of a polynomial ring $W\left[X_{1}, \ldots, X_{d}\right]$ at a maximal ideal of the type $\left(\pi, f\left(X_{1}\right), X_{2}, \ldots, X_{d}\right)$ where $f$ is a monic irreducible polynomial in $W\left[X_{1}\right]$ and $(W, \pi)$ is an excellent d.v.r. $\subset A$; moreover $A$ is an étale neighborhood of $B$.

(2) There exists an element $h$ in $B \cap a A$ such that $B / h B \rightarrow A / a A$ is an isomorphism. Furthermore $h A=a A$.

Proof. The first part consists of arguments similar to those in the proof of the local version of Zariski's Main Theorem in [N]. Let $y_{1}, \ldots, y_{t}$ be elements of $A$ such that their residue classes $\bar{y}_{1}, \ldots, \bar{y}_{t}$ modulo $m$ form a transcendence basis of $A / m$ over $V / \pi V$. Write $L=V / \pi V\left(\bar{y}_{1}, \ldots, \bar{y}_{t}\right)$ and $W=V\left[y_{1}, \ldots, y_{t}\right]_{\left(\pi V\left[y_{1}, \ldots, y_{t}\right]\right)}$ (since $V$ is a d.v.r. $y_{1}, \ldots, y_{t}$ are algebraically independent over $V$ ); note $W / \pi W=L$.

Consider $T=W\left[a, X_{2}, \ldots, X_{d}\right]$ where $\pi, X_{2}, \ldots, X_{d}$ form a part of a regular system of parameters of $A$. Then $a, X_{2}, \ldots, X_{d}$ are algebraically independent over $W$. By the altitude formula $A$ is algebraic over $T$. Let $C$ denote the integral closure of $T$ in $A$ and write $m_{1}=m \cap C$. Then $C$ is a finite $T$ module and $A=C_{m_{1}}$ (Zariski's Main Theorem).

By our construction $C / m_{1}=K=L(\bar{\theta})$ for some $\theta$ in $C$. Let $\bar{f}$ denote the monic irreducible polynomial of $\bar{\theta}$ over $L$. Let $f$ lift $\bar{f}$ over $W$. Then $f$ is monic irreducible over $W$ and $f(\theta) \in m_{1}$, but $f^{\prime}(\theta) \notin m_{1}$. Note that, changing $\theta$ to $\theta+\alpha, \alpha \in m_{1}$, if necessary, we can assume that $\pi, f(\theta), X_{2}, \ldots, X_{d}$ generate $m$ in $A$. Since $C$ is semi-local, we can choose $X_{1}$ in $C$ such that $X_{1} \equiv \theta \bmod m^{2}$ and the ideal $\left(\pi, a, f\left(X_{1}\right), X_{2}, \ldots, X_{d}\right)$ is contained in $m_{1}$ only. It follows easily that $m=\left(\pi, f\left(X_{1}\right), X_{2}, \ldots, X_{d}\right)$.

As $X_{1}$ is integral over $T$, replacing $X_{1}$ by $X_{1}+a^{i}$ for $i$ sufficiently large we can assume that $a$ is integral over $W\left[X_{1}, \ldots, X_{d}\right]$. Let $D=W\left[X_{1}, \ldots, X_{d}\right], q=$ $\left(\pi, f\left(X_{1}\right), X_{2}, \ldots, X_{d}\right)$ and $B=D_{q}$. Note that $C$ is a finite $D[a]$ module, $C / m_{1}=$ $D[a] /(q, a)$. Hence $A=C_{m_{1}}=D[a]_{(q, a)}$. By our construction of $B, m_{B} A=m$; $B / m_{B}=A / m$; hence $i: B \rightarrow A$ is unramified, but $B$ being regular, $i$ is étale. Hence $B \cap a A$ is principal. Let $B \cap a A=(h)$. It is easy to check that $h A=a A$; hence $B / h B \simeq A / a A$.

We begin this section by stating Serre's conjecture on intersection multiplicity.

Serre's Conjecture. Let $R$ be a regular local ring and let $M, N$ be two finitely generated modules over $R$ such that $\ell\left(M \otimes_{R} N\right)<\infty$. Then

$$
\chi(M, N) \quad\left(=\Sigma(-1)^{i} \ell\left(\operatorname{Tor}_{i}^{R}(M, N)\right)\right)
$$


is $\geq 0$, the sign of equality holds if and only if $\operatorname{dim} M+\operatorname{dim} N<\operatorname{dim} R$.

Serre proved the conjecture for the equicharacteristic and the unramified case. The vanishing part of the conjecture was proved by Roberts $[R]$ and by Gillet and Solue $[\mathrm{G}-\mathrm{S}]$ independently. The positivity part is very much open. For some special cases of positivity see $[\mathrm{D} 2, \mathrm{~K}], \mathrm{M}]$.

For our proof of Serre's theorem, we need the following observations.

2.1 Lemma ( $\overline{\mathrm{D} 2})$. Let $(A, m)$ be a local domain essentially of finite type over a field $K$. Let $P$ be a prime ideal of $A$. Then there exists a local domain $(B, n)$ essentially of finite type over some field $L \subset K$ and a prime ideal $q$ of $B$ such that

(i) $(B, n) \rightarrow(A, m)$ is local, flat and $\operatorname{dim} B=\operatorname{dim} A$.

(ii) $B / n$ is separately generated over $L$.

(iii) $q A=P$.

Proof. If $A / m$ is separately generated over $K$, there is nothing to prove. So, assume this is not so. Then obviously $K$ is not algebraic over its prime field $k$. Now following blindly the technique introduced by Peskine and Szpiro [P-S, Chapter II, Lemma (2.2), Lemma (2.3) and Lemma (2.4)] we can construct an infinite field $L$, $k \subset L \subset K$ and the local domain $(B, n)$ with the above properties. We leave the details as an exercise to the reader.

Remark. If $(A, m)$ is regular, then so is $(B, n)$.

2.2 Lemma. Let $\left(A, m_{A}\right)$ and $\left(A^{\prime}, m_{A^{\prime}}\right)$ be two regular local rings with $\operatorname{dim} A=$ $\operatorname{dim} A^{\prime}$ and let $f: A \rightarrow A^{\prime}$ be local and flat. Let $M, N$ be two finitely generated modules over $A$ such that $\ell(M \otimes N)<\infty$. Write $M^{\prime}=M \otimes_{A} A^{\prime}, N^{\prime}=N \otimes_{A}$ $A^{\prime}$. Then $\ell\left(M^{\prime} \otimes_{A^{\prime}} N^{\prime}\right)<\infty$ and $\chi^{A}(M, N)=\ell\left(A^{\prime} / m_{A^{\prime}}\right) \chi^{A^{\prime}}\left(M^{\prime}, N^{\prime}\right)$. Thus $\chi^{A}(M, N) \gtreqless 0$ according as $\chi^{A}\left(M^{\prime}, N^{\prime}\right) \lesseqgtr 0$ respectively.

Proof. Proof is obvious - and we leave it as an exercise.

2.3 Lemma. Let $V \stackrel{f}{\rightarrow} A$ be non-zero, local, smooth map, where $A$ is a regular local ring and $V$ is a d.v.r. or a field. Then we can construct a commutative diagram

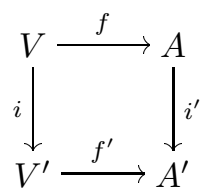

such that $V^{\prime}$ is a d.v.r. or a field, $A^{\prime}$ is a regular local ring, $i$ is flat, $m_{V} V^{\prime}=m_{V^{\prime}}$ and $V^{\prime} / m_{V^{\prime}}$ is algebraically closed. Moreover, $f^{\prime}$ is smooth and $i^{\prime}$ is flat.

Proof. For construction of $V^{\prime}$ and $i$, we refer the reader to Mat. Now localizing at a maximal ideal of $V^{\prime} \otimes_{V} A$, lying over $m_{A}$, we get our required $A^{\prime}$. All other things can be checked easily.

Note that, by completing $V^{\prime}$ if necessary, we can assume $V^{\prime}$ is excellent.

Now we are ready to give a new proof of the geometric case of Serre's Theorem on Intersection Multiplicity.

2.4 Theorem ([S, Serre, Th. 1, Th. 2, Ch. V]). Let $A$ be a regular local ring essentially of finite type over a field $k$ or an excellent d.v.r. $V$. In the d.v.r. case we assume $i: V \rightarrow R$ is unramified (smooth). Let $M$ and $N$ be two finitely generated 
modules with $\ell\left(M \otimes_{A} N\right)<\infty$. Write $\chi(M, N)=\sum_{i=0}^{\operatorname{dim} A}(-1)^{i} \ell\left(\operatorname{Tor}_{i}^{A}(M, N)\right)$. Then $\chi(M, N) \geq 0$, the sign of equality holds if and only if $\operatorname{dim} M+\operatorname{dim} N<\operatorname{dim} A$.

Proof. Without any loss of generality, we can assume $M=A / P, N=A / \mathfrak{q}$, where $P, \mathfrak{q}$ are prime ideals of $A$. If $A$ is essentially of finite type over $k$, then by Lemma 2.1, we can assume $A / m_{A}$ is separately generated over $k$. Thus $V$ (resp. $k) \hookrightarrow A$ is smooth. By lifting a transcendental basis of $A / m_{A}$ over $V / m_{V}$ (resp. $k$ ), and then localizing (first part of proof of Theorem 1.3) we can assume $A / m_{A}$ is algebraic over $V / m_{V}$ (resp. $k$ ). By Lemma 2.2 and Lemma 2.3, we can assume that $V / m_{V}$ (resp. $k$ ) is algebraically closed and hence is isomorphic to $A / m_{A}$.

Choose a non-null element $a$ in $P \cap \mathfrak{q} \cap m_{A}^{2}$. Then by Theorem 1.3 we can find $X_{1}, \ldots, X_{d}$ in $A, d=\operatorname{dim} A$, such that if $B=V\left[X_{1}, \ldots, X_{d}\right]\left(\right.$ resp. $\left.k\left[X_{1}, \ldots, X_{d}\right]\right)$, $n=m \cap B=\left(\pi, f\left(X_{1}\right), X_{2}, \ldots, X_{d}\right)$ (resp. $\left.\left(f\left(X_{1}\right), X_{2}, \ldots, X_{d}\right)\right)$ then $A$ is a étale neighborhood of $B_{n}$ and $B_{n} /(a) \cap B_{n} \hookrightarrow A / a A$ is an isomorphism. (Here $(\pi)=m_{V}$.) Actually $a A=\left((a) \cap B_{n}\right) A$. Since $V / m_{V}$ (resp. $k$ ) is algebraically closed, $n=\left(\pi, X_{1}-\lambda, X_{2}, \ldots, X_{d}\right)$, (resp. $\left(X_{1}-\lambda, X_{2}, \ldots, X_{d}\right)$. So, by a translation, if necessary, we can assume $n=\left(\pi, X_{1}, X_{2}, \ldots, X_{d}\right)$ (resp. $\left.\left(X_{1}, \ldots, X_{d}\right)\right)$. It is easy to check that if $P^{\prime}=P \cap B_{n}$ and $\mathfrak{q}^{\prime}=\mathfrak{q} \cap B_{n}$, then $P^{\prime} A=P$, $\mathfrak{q}^{\prime} A=\mathfrak{q}$ and $\chi^{B_{n}}\left(B_{n} / P^{\prime}, B_{n} / \mathfrak{q}^{\prime}\right)=\chi^{A}(A / P, A / \mathfrak{q})$. Thus we can assume $A=$ $V\left[X_{1}, \ldots, X_{d}\right]_{\left(\pi, X_{1} \ldots X_{d}\right)}\left(\right.$ resp. $\left.k\left[X_{1}, \ldots, X_{d}\right]_{\left(X_{1}, X_{2}, \ldots, X_{d}\right)}\right)$, and $P, \mathfrak{q}$ are two prime ideals of $A$ such that $\ell(A / P+\mathfrak{q})<\infty$. Note that the technique of diagonalization works on a polynomial ring localized at the maximal ideal $\left(\pi, X_{1}, \ldots, X_{d}\right)$ (resp. $\left.\left(X_{1} \ldots X_{d}\right)\right)$. Write $C=k\left[X_{1} \ldots Y_{n}, X_{1} \ldots Y_{n}\right]_{\left(X_{1}, \ldots, X_{n}, Y_{1}, \ldots, Y_{n}\right)}$, or $C=$ $V\left[X_{1}, \ldots, X_{n}, Y_{1}, \ldots, Y_{n}\right]_{\left(\pi, X_{1}, \ldots, X_{n}, Y_{1}, \ldots, Y_{n}\right)}, T=C / P C+\mathfrak{q} C$ and $D=\left(X_{1}-\right.$ $\left.Y_{1}, \ldots, X_{n}-Y_{n}\right)$. Then in the first case $\operatorname{Tor}_{i}^{A}(A / P, A / \mathfrak{q}) \simeq \operatorname{Tor}_{i}^{C}(C / P C+\mathfrak{q} C, C / D)$ and in the second case $\operatorname{Tor}_{i}^{C}\left(\operatorname{Tor}_{j}^{V}(A / P, A / \mathfrak{q}), C / D\right) \Rightarrow \operatorname{Tor}_{i+j}^{A}(A / P, A / \mathfrak{q})$; here $j=0$ or 1 . In the latter case we need to separate the cases for $\pi \notin A / P$ and for $\pi \in A / P$ and $\pi \in A / \mathfrak{q}$ as discussed in [S] (page 140). Thus we are done by similar arguments as in [S].

We now proceed to cover the complete case i.e. when $A=V\left[\left[X_{1}, \ldots, X_{d}\right]\right]$, where $V$ is a complete d.v.r. or a field $k$. Since the same technique will work for both cases, we will work out the case when $V=k$, a field. In this case we write $\hat{A}=k\left[\left[X_{1}, \ldots, X_{d}\right]\right], A=k\left[X_{1}, \ldots, X_{d}\right]_{\left(X_{1}, \ldots, X_{d}\right)}, A^{h}=$ the henselization of $A$. Recall that $A^{h}=\underset{n \in I}{\lim _{n \rightarrow I}} A_{n}$, where each $A_{n}$ is an étale neighborhood of $A$ and hence a regular local ring, essentially of finite type over $k$. We know, by Artin's Approximation Theorem, that $A^{h}$ is an approximation ring i.e. given any system of equations $F_{1}=0, \ldots, F_{\gamma}=0, F_{i} \in A\left[X_{1}, \ldots, X_{d}\right]$, if the system has a solution $\hat{x}_{1}, \ldots, \hat{x}_{d}$ in $\hat{A}$, then for every $t>0, \exists$ a solution $x_{1}^{(t)}, \ldots, x_{1}^{(t)}$ of the system in $A^{h}$ such that $x_{i}^{(t)} \equiv \hat{x}_{i} \bmod \hat{m}^{t} \forall i=1, \ldots, \ell$. Since for any such system of equations, any solution consists of finite number of elements, we can always assume that these $x_{i}^{(t)}$ 's lie in $A_{n}$ for some $n \in I$. We will utilize this fact to reduce our problem to a similar problem for a regular local ring essentially of finite type over $k$. We achieve this by the following sequence of results.

We start with a theorem and a corollary, both due to Peskine and Szpiro. 
3.1 Theorem ([$[\mathrm{P}-\mathrm{S}, 6.2])$. Let $V$ be a field or an excellent d.v.r., and let $A$ be a local ring essentially of finite type over $V$. Let $\hat{A}$ denote the completion of $A$ with respect to the maximal ideal $m$ of $A$. Let $M$ be a finitely generated module of finite projective dimension. Consider a minimal free resolution of $M$ over $\hat{A}$ :

$$
0 \rightarrow L_{r} \rightarrow L_{r-1} \rightarrow \cdots \rightarrow L_{1} \rightarrow L_{0} \rightarrow M \rightarrow 0 .
$$

Then, for every integer $c$, there exists an exact complex:

$$
0 \rightarrow P_{r} \rightarrow P_{r-1} \rightarrow \cdots \rightarrow P_{1} \rightarrow P_{0}
$$

of finitely generated free modules over $A^{h}$ such that $P_{\bullet} \otimes_{A^{h}}\left(A^{h} / m^{c} A^{h}\right)=L_{\bullet} \otimes_{\hat{A}}$ $\left(\hat{A} / m^{c} \hat{A}\right)$

Corollary ( $[\mathrm{P}-\mathrm{S}, 6.3])$. Let $A, M$ be as above. Then for every integer $c$, there exists an $n \in I$ and an $A_{n}$-module $M_{n}$ of finite projective dimension such that

(i) $M_{n} / m^{c} M_{n} \simeq M / m^{c} M$.

(ii) $P d_{A_{n}} M_{n}=P d_{\hat{A}} M$.

For proof, we refer the reader to $[\mathrm{P}-\mathrm{S}]$. Note that the complex $P_{\bullet}$, in the theorem, can also be considered as a complex over $A_{n}$, for some $n \in I$. (We now return to our notations described in the beginning of this section.)

3.2.1 Lemma. Let $\hat{M}$ be a module of finite length over $\hat{A}$. Then there exists a module $M_{n}$ of finite length over some $A_{n}$, such that $M_{n} \otimes_{A_{n}} \hat{A}=\hat{M}$.

Proof. We know that $\hat{M}$ itself, considered as an $A_{n}$-module, can do the job. Here we want to take a different approach, which will be useful in several occasions (Remark at the end of this proof). Let $t$ be an integer such that $\hat{m}^{t} \hat{M}=0$. Suppose $r=\mu(\hat{M})$. Now we construct the following commutative diagram with exact horizontal rows:

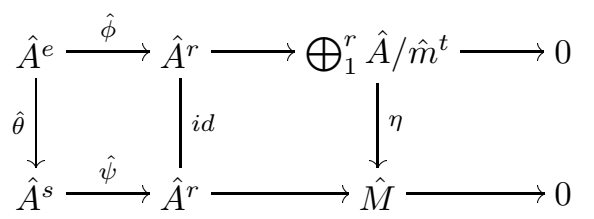

where $\eta$ is the natural surjection, sending $\bar{e}_{i}$ to $z_{i}$ where $z_{1}, \ldots, z_{r}$ is a set of minimal generators of $\hat{M}, \hat{\phi}=\left(a_{i j}\right)_{r \times e},\left(a_{i j}\right)$ constructed from a minimal set of generators of $m^{t}, \hat{\psi}=\left(\hat{b}_{i j}\right)_{r \times s}$ and $\hat{\theta}=\left(\hat{c}_{i j}\right)_{s \times e}$, such that $\hat{\phi}=\hat{\psi} \hat{\theta}$. Let us consider the variable matrices $\left(X_{i j}\right),\left(Y_{i j}\right)$ and $\left(Z_{i j}\right)$ corresponding to $\hat{\phi}, \hat{\psi}$ and $\hat{\theta}$ respectively. Then $\left(\hat{a}_{i j}\right),\left(\hat{b}_{i j}\right)$ and $\left(\hat{c}_{i j}\right)$ are solutions of the following system of equations

$$
X_{i j}=a_{i j} \quad \text { for every } i, j \quad \text { and } \quad\left(Y_{i j}\right)\left(Z_{i j}\right)=\left(X_{i j}\right), \ldots
$$

(in other words, the $a_{i j}$ 's play the role of coefficients in the above system of equations) we approximate these solutions modulo $\hat{m}^{t}$ by $\phi=\left(a_{i j}\right), \psi=\left(b_{i j}\right)$ and $\theta=\left(c_{i j}\right)$, lying in some $A_{n}$, respectively. 
Now consider the following commutative diagram with exact horizontal rows:

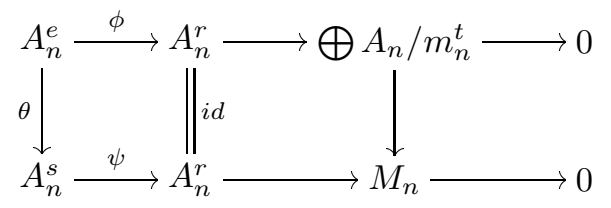

Write $M_{n}$ for coker $\psi$. Then it follows from (3) that $m_{n}^{t} M_{n}=0$ and hence $\ell\left(M_{n}\right)<$ $\infty$. Since $\psi \equiv \hat{\psi} \bmod \hat{m}^{t}$, and $\hat{m}^{t} \hat{M}=0$ it follows easily that $M_{n} \otimes_{A_{n}} \hat{A}=\hat{M}$.

3.2.2 Lemma. Let $\hat{M}, \hat{N}$ be two finitely generated modules over $\hat{A}$ with minimal free resolutions $\hat{F}_{\bullet}, \hat{G}_{\bullet}$ respectively. Suppose that $\ell\left(\hat{M} \otimes_{\hat{A}} \hat{N}\right)<\infty$. Then there exists finitely generated modules $M_{n}$ and $N_{n}$ with minimal free resolutions $F_{\bullet}$, $G_{\bullet}$ over some $A_{n}$ such that the conclusions of 3.1 are satisfied for $F_{\bullet}, G_{\bullet n}$ and $\ell\left(M_{n} \otimes_{A_{n}} N_{n}\right)<\infty$.

Proof. Construction of $F_{\bullet}, G_{\bullet n}$ over any $A_{n}$ follows from Theorem 3.1 and its corollary. To ensure that $\ell\left(M_{n} \otimes_{A_{n}} N_{n}\right)$ is finite we add equations arising from (1), as in Lemma 3.2.1, where $\hat{M}$ is replaced by $\hat{M} \otimes_{\hat{A}} \hat{N}$. The approximations of solutions of these equations along with the equations for $\hat{F}_{\bullet}$ and $\hat{G}_{\bullet}$ determine the required $A_{n}, M_{n}$ and $N_{n}$.

3.3 Proposition. Let $0 \rightarrow \hat{N} \rightarrow \hat{M} \rightarrow \hat{T} \rightarrow 0$ be an exact sequence of finitely generated $\hat{A}$-modules and let $\hat{F}_{\bullet}, \hat{L}_{\bullet} \hat{G}_{\bullet}$ be free resolutions of $\hat{N}, \hat{M}, \hat{T}$ respectively where $\hat{F}_{\bullet}, \hat{G}_{\bullet}$ are minimal and $\hat{L}_{\bullet}=\hat{F}_{\bullet} \oplus \hat{G}_{\bullet}$ is constructed from $\hat{F}_{\bullet}, \hat{G}_{\bullet}$ in the usual manner i.e. if $\hat{\phi}_{\bullet}, \hat{\psi}_{\bullet}$ represents the boundary maps of $\hat{F}_{\bullet}, \hat{G}_{\bullet}$ respectively; and $\hat{h}_{\bullet}$ represents the map from $\left(\hat{G}_{\bullet}\right)_{1} \rightarrow F_{\bullet}$ with $\hat{h}_{n} \hat{\psi}_{n+1}+\hat{\phi}_{n} \hat{h}_{n+1}=0$, then $\hat{\theta}_{\bullet}=\left(\begin{array}{ll}\hat{\phi}_{\bullet} & \hat{h}_{\bullet} \\ 0 & \hat{\psi}_{\bullet}\end{array}\right)$ represents the boundary maps of $L_{\bullet}$. Then for every positive integer $t$, there exists an $n \in I$ and an exact sequence of finitely generated $A_{n}$-modules

$$
0 \rightarrow N_{n} \rightarrow M_{n} \rightarrow T_{n} \rightarrow 0
$$

such that

(i) $N_{n} \otimes A_{n} / m_{n}^{t}=\hat{N} \otimes \hat{A} / \hat{m}^{t}, M_{n} \otimes A_{n} / m_{n}^{t}=\hat{M} \otimes \hat{A} / \hat{m}^{t}, T_{n} \otimes A_{n} / m_{n}^{t}=$ $\hat{T} \otimes \hat{A} / \hat{m}^{t}$.

(ii) There exists free resolutions $F_{\bullet}, L_{\bullet}, G_{\bullet}$ of $N_{n}, M_{n}$ and $T_{n}$ respectively such that

(a) $F_{\bullet}, G_{\bullet}$ are minimal and $L_{\bullet}=F_{\bullet} \oplus G_{\bullet}$ is constructed in the usual manner,

(b) $F_{\bullet} \otimes A_{n} / m_{n}^{t}=\hat{F} \bullet \otimes \hat{A} / \hat{m}^{t}, L \bullet \otimes A_{n} / m_{n}^{t}=\hat{L} \bullet \otimes \hat{A} / \hat{m}^{t}$ and $G \bullet \otimes A_{n} / m_{n}^{t}=$ $\hat{G}_{\bullet} \otimes \hat{A} / \hat{m}^{t}$.

Proof. Write $\hat{\phi}_{\bullet}=\left(\hat{a}_{i j}\right), \hat{\psi}_{\bullet}=\left(\hat{b}_{i j}\right), \hat{h}_{\bullet}=\left(\hat{h}_{i j}\right)$.

Treating the entries of $\hat{\phi}_{\bullet}, \hat{\psi}_{\bullet}$ and $\hat{h}_{\bullet}$ as variables we see that $\hat{a}_{i j}, \hat{b}_{i j}$ and $\hat{h}_{i j}$ satisfy equations

$$
\hat{\phi}_{i} \cdot \hat{\phi}_{i+1}=0, \hat{\psi}_{i} \cdot \hat{\psi}_{i+1}=0 \quad \text { and } \quad \hat{\theta}_{i} \cdot \hat{\theta}_{i+1}=0 \quad\left(\text { or } \quad \hat{h}_{n} \hat{\psi}_{n+1}+\hat{\phi}_{n} \hat{h}_{n+1}=0\right) .
$$

Let $a_{i j}, b_{i j}, h_{i j}$ denote the solutions of the above system in some $A_{n}$ such that they approximate $\hat{a}_{i j}, \hat{b}_{i j}, \hat{h}_{i j}$ modulo $\hat{m}^{t}$ respectively. Write $\phi_{\bullet}=\left(a_{i j}\right), \psi_{\bullet}=$ $\left(b_{i j}\right), h_{\bullet}=\left(h_{i j}\right)$ and $\theta_{\bullet}=\left(\begin{array}{ll}\phi_{\bullet} & h_{\bullet} \\ 0 & \psi_{\bullet}\end{array}\right)$. 
Let us form $F_{\bullet}, L_{\bullet}$ and $G_{\bullet}$ in the expected manner (i.e., $L_{\bullet}=F_{\bullet} \oplus G_{\bullet}$ etc. with boundary maps $\phi_{\bullet}, \theta_{\bullet}, \psi_{\bullet}$ respectively). Write $N_{n}=$ coker $\phi_{1}, M_{n}=$ coker $\theta_{1}$, and $T_{n}=$ coker $\psi_{1}$. Now (i) and (ii) follow from Theorem (3.1) and its corollary directly. Also from the construction it can be easily checked that

$$
0 \rightarrow N_{n} \rightarrow M_{n} \rightarrow T_{n} \rightarrow 0
$$

is exact.

Remarks. 1. If $M$ is free, so is $M_{n}$.

2. Using the above proposition we can prove the following: Given a finite complex

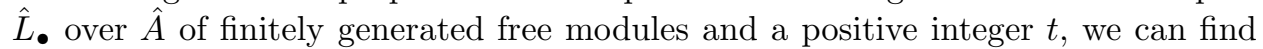
$n \in I$ and a finite complex $L_{\bullet}$ of finitely generated free modules over $A_{n}$ such that

(i) $\hat{L} \bullet \otimes \hat{A} / \hat{m}^{t} \simeq L_{\bullet n} \otimes A_{n} / m_{n}^{t}$ and

(ii) $H_{i}\left(\hat{L}_{\bullet}\right) \otimes \hat{A} / \hat{m}^{t} \simeq H_{i}\left(L_{\bullet}\right) \otimes A_{n} / m_{n}^{t}$.

Thus, when all the homologies of $\hat{F}_{\bullet}$ are of finite length, for $t>>0, \exists n \in I$ such that $H_{i}\left(\hat{L}_{\bullet}\right) \simeq H_{i}\left(L_{\bullet} n\right)$ for $i \geq 0$. But, when $\hat{L}_{\bullet}=\hat{F}_{\bullet} \otimes \hat{G}_{\bullet}$ where $\hat{F}_{\bullet}, \hat{G}_{\bullet}$ are two minimal free complexes resolving $\hat{M}, \hat{N}$ respectively, (notations as in 3.2) $L_{\bullet}$ is not necessarily $F_{\bullet n} \otimes G_{\bullet}$.

Theorem 3.4. Let $\hat{M}, \hat{N}$ be two finitely generated modules over $\hat{A}$. Then for every integer $t$, there exists an $n \in I$ and two finitely generated $A_{n}$-modules $M_{n}, N_{n}$ such that

(i) both $M_{n}$ and $N_{n}$ satisfy Corollary (3.1) and

(ii) $\operatorname{Tor}_{i}^{A_{n}}\left(M_{n}, N_{n}\right) \otimes A_{n} / m_{n}^{t} \simeq \operatorname{Tor}_{i}^{\hat{A}}(\hat{M}, \hat{N}) \otimes \hat{A} / \hat{m}^{t}$.

Proof. Given a positive integer $t$, the construction of $M_{n}, N_{n}$ and the assertion in part (ii) for $i=0$, follow directly from (3.1). Now, consider the following short exact sequence

$$
0 \rightarrow \hat{T} \rightarrow \hat{A}^{r} \rightarrow \hat{M} \rightarrow 0
$$

By Proposition (3.3), there exists a short exact sequence of $A_{n}$-modules

$$
0 \rightarrow T_{n} \rightarrow A_{n}^{r} \rightarrow M_{n} \rightarrow 0
$$

such that $\hat{T} / \hat{m}^{t} \hat{T}=T_{n} / m_{n}^{t} T_{n}$ and part (ii) of Proposition (3.3) is satisfied. Hence, it is enough to prove our assertion for Tor 1 . Applying $\otimes \hat{A} / \hat{m}^{t}$ to (1) and $\otimes A_{n} / m_{n}^{t}$ to $\left(1^{\prime}\right)$ respectively we obtain the following exact sequences

$$
0 \rightarrow \operatorname{Tor}_{1}^{\hat{A}}\left(\hat{M}, \hat{A} / \hat{m}^{t}\right) \rightarrow \hat{T} / m^{t} \hat{T} \rightarrow \bigoplus_{1}^{r} \hat{A} / \hat{m}^{t} \rightarrow \hat{M} / \hat{m}^{t} \hat{M} \rightarrow 0
$$

and

$$
0 \rightarrow \operatorname{Tor}_{1}^{A_{n}}\left(M_{n}, A_{n} / m_{n}^{t}\right) \rightarrow T_{n} / m_{n}^{t} T_{n} \rightarrow \bigoplus_{1}^{r} A_{n}^{r} / m_{n}^{t} \rightarrow M_{n} / m_{n}^{t} M_{n}-0 .
$$

By the Five-Lemma, it follows that $\operatorname{Tor}_{1}^{\hat{A}}\left(\hat{M}, \hat{A} / \hat{m}^{t}\right) \simeq \operatorname{Tor}_{1}^{A_{n}}\left(M_{n}, A_{n} / m_{n}^{t}\right)$ and hence $\operatorname{Tor}_{i}^{\hat{A}}\left(\hat{M}, \hat{A} / \hat{m}^{t}\right) \simeq \operatorname{Tor}_{i}^{A_{n}}\left(M_{n}, A_{n} / m_{n}^{t}\right)$ for $i \geq 0$. It is an easy exercise to notice that similar arguments establish the following: $\operatorname{Tor}_{i}^{\hat{A}}\left(\hat{M} \otimes \hat{N}, \hat{A} / \hat{m}^{t}\right) \simeq$ $\operatorname{Tor}_{i}^{A_{n}}\left(M_{n} \otimes N_{n}, A_{n} / m_{n}^{t}\right)$ for $i \geq 0$.

Again applying $\otimes \hat{N}$ to (1) and $\otimes N_{n}$ to $\left(1^{\prime}\right)$ respectively we obtain the following short exact sequences 
(A): $\quad 0 \rightarrow \operatorname{Tor}_{1}^{\hat{A}}(\hat{M}, \hat{N}) \rightarrow \hat{T} \otimes \hat{N} \rightarrow \hat{Q} \rightarrow 0 ; 0 \rightarrow \hat{Q} \rightarrow \hat{N}^{r} \rightarrow \hat{M} \otimes \hat{N} \rightarrow \ldots$

(B): $\quad 0 \rightarrow \operatorname{Tor}_{1}^{A_{n}}\left(M_{n}, N_{n}\right) \rightarrow T_{n} \otimes N_{n} \rightarrow Q_{n} \rightarrow 0,0 \rightarrow Q_{n} \rightarrow N_{n}^{r} \rightarrow M_{n} \otimes$ $N_{n} \rightarrow 0$.

Tensoring the second set of exact sequences of (A) and (B) by $\hat{A} / \hat{m}^{t}$ and $A_{n} / m_{n}^{t}$ respectively and applying the Five-Lemma we get

$$
\operatorname{Tor}_{i}^{\hat{A}}\left(\hat{Q}, \hat{A} / \hat{m}^{t}\right) \simeq \operatorname{Tor}_{i}^{A_{n}}\left(Q_{n}, A_{n} / m_{n}^{t}\right)
$$

for $i \geq 0$. Now tensoring the first set of exact sequences of (A) and (B) by $\hat{A} / \hat{m}^{t}$ and $A_{n} / m_{n}^{t}$ respectively, by the Five-Lemma we obtain, for $j \geq 0$.

$$
\operatorname{Tor}_{j}^{\hat{A}}\left(\operatorname{Tor}_{1}^{\hat{A}}(\hat{M}, \hat{N}), \hat{A} / \hat{m}^{t}\right) \simeq \operatorname{Tor}_{j}^{A_{n}}\left(\operatorname{Tor}_{1}^{A_{n}}\left(M_{n}, N_{n}\right), A_{n} / m_{n}^{t}\right) .
$$

Hence we are done.

Corollary. If $\ell(\hat{M} \otimes \hat{N})<\infty$, then $\exists$ an $n \in I$ and two finitely generated modules $M_{n}, N_{n}$ over $A_{n}$ such that

(i) $\ell\left(M_{n} \otimes N_{n}\right)<\infty$ and

(ii) $\operatorname{Tor}_{i}^{A_{n}}\left(M_{n}, N_{n}\right) \otimes_{A_{n}} \hat{A} \simeq \operatorname{Tor}_{i}^{\hat{A}}(\hat{M}, \hat{N})$.

The proof follows directly from the above theorem and Lemma (3.2.2), by taking $t>>0$, so that $\hat{m}^{t}(\hat{M} \otimes \hat{N})=0$.

3.5 Proposition. Let $\hat{M}$ be a finitely generated $\hat{A}$-module with $\operatorname{dim} \hat{M}=d$. Then $\exists$ a module $M_{n}$ over $A_{n}$ for some $n \in I$ such that $\operatorname{dim} M_{n}=d$ and $M_{n} \otimes \hat{A} / \hat{m}^{t}=$ $\hat{M} / m^{t} \hat{M}$.

Proof. First note that by using arguments as in Lemma (3.2.1) if $x_{1}, \ldots, x_{d}$ is a maximal $\hat{A}$-sequence in $\hat{m}$, we can approximate it, by a maximal $A_{n}$-sequence $y_{1}, \ldots, y_{d}$ in $A_{n}$, for some $n$, modulo $\hat{m}^{t}$, if $\hat{m}^{t}$ annihilates $\hat{A} /\left(x_{1}, \ldots, x_{d}\right)$. Thus any regular sequence $x_{1}, \ldots, x_{r}$ in $\hat{A}$ can be approximated by a regular sequence $y_{1}, \ldots, y_{r}$ in $A_{n}$ for some $n \in I$, (extend it to maximal $\hat{A}$-sequence!). Let $x_{1}, \ldots, x_{r}$ be an $\hat{A}$ sequence which is also a system of parameters for $\hat{M}$. So $\ell\left(\hat{M} /\left(x_{1}, \ldots, x_{r}\right) \hat{M}\right)<\infty$. Then, by the above proposition, we can approximate $\hat{M}$ and $\hat{A} /\left(x_{1}, \ldots, x_{r}\right) \hat{A}$ by $M_{n}$ and $A_{n} /\left(y_{1}, \ldots, y_{r}\right)$ respectively such that

$$
\begin{gathered}
M_{n} \otimes A_{n} / m_{n}^{t} \simeq \hat{M} \otimes \hat{A} / \hat{m}^{t}, A_{n} /\left(y_{1}, \ldots y_{r}, m_{n}^{t}\right) \simeq \hat{A} /\left(x_{1}, \ldots, x_{r}, \hat{m}^{t}\right), \\
\operatorname{Tor}_{i}^{A_{n}}\left(M_{n}, A_{n} /\left(y_{1}, \ldots, y_{r}\right)\right) \otimes_{A_{n}} \hat{A}=\operatorname{Tor}_{i}^{\hat{A}}\left(\hat{M}, \hat{A} /\left(x_{1}, \ldots x_{r}\right) \hat{A}\right),
\end{gathered}
$$

and $\ell\left(M_{n} \otimes A_{n} /\left(y_{1}, \ldots, y_{r}\right)\right)<\infty$. Thus

$$
\chi^{A_{n}}\left(M_{n}, A_{n} /\left(y_{1}, \ldots, y_{r}\right)\right)=\chi^{\hat{A}}\left(\hat{M}, \hat{A} /\left(x_{1}, \ldots, x_{r}\right)\right) .
$$

This implies $\left[\mathrm{L}\right.$ that, $\operatorname{dim} M_{n}=\operatorname{dim} \hat{M}$.

Remark. We also have a different proof of the above fact using 3.3 only.

3.6 Theorem. Let $\hat{M}, \hat{N}$ be two finitely generated modules over $\hat{A}$ such that $\ell(\hat{M} \otimes \hat{N})<\infty$. Then $\chi(\hat{M}, \hat{N}) \geq 0$, the sign of equality holds if and only if $\operatorname{dim} \hat{M}+\operatorname{dim} \hat{N}<\operatorname{dim} \hat{A}$.

Proof. By the corollary to Theorem 3.4 and Proposition 3.5, we can approximate $\hat{M}, \hat{N}$ by $M_{n}, N_{n}$ over $A_{n}$, for some $n \in I$, such that 
(i) $\operatorname{dim} M_{n}=\operatorname{dim} \hat{M}, \operatorname{dim} N_{n}=\operatorname{dim} \hat{N}, M_{n} \otimes A_{n} / m_{n}^{t} \simeq \hat{M} \otimes \hat{A} / \hat{m}^{t}, N_{n} \otimes$ $A_{n} / m_{n}^{t} \simeq \hat{M} \otimes \hat{A} / \hat{m}^{t}$ and

(ii) $\operatorname{Tor}_{i}^{A_{n}}\left(M_{n}, N_{n}\right) \otimes_{A_{n}} \hat{A} \simeq \operatorname{Tor}_{i}^{\hat{A}}(\hat{M}, \hat{N})$.

Thus $\chi^{A_{n}}\left(M_{n}, N_{n}\right) \simeq \chi^{\hat{A}}(\hat{M}, \hat{N})$ and the dimension considerations are identical for both pairs. Since $A_{n}$ is a regular local ring essentially of finite type over $k$ (smooth over $V$ ), we are done by Theorem 2.4.

4

Here we sketch proofs of Quillen's theorem on Chow groups and its extension by Gillet and Levine. As in the previous sections the arguments we give are in part based upon our generalization (1.3) of the Lindel [Li], Nashier Nas] Theorems. One should consult [D2] for more details.

4.1 Theorem (Quillen). Let $(A, m)$ be a regular local ring essentially of finite type over a field $k$. Then $\mathbb{A}_{i}(A)=0$ for $0 \leq i<\operatorname{dim} A$.

Proof. Let $P$ be a prime ideal of height $\operatorname{dim} A-i$. By Lemma 2.1 we can assume that $A / m$ is separably generated over $k$. Choose $a \neq 0 \in P \cap m^{2}$. Now construct $(B, n)$ as in Theorem 1.3. By the work of Claborn and Fossum $[\mathrm{C}-\mathrm{F}$ ] we have $\mathbb{A}_{i}(B)=0$ for $0 \leq i<\operatorname{dim} A$. Since $B \rightarrow A$ is flat and by $(P \cap B) A=P$, we are done.

4.2 Theorem (Gillet-Levine). Let $(A, m, K)$ be a regular local ring, essentially of finite type and smooth over an excellent d.v.r. $V$ such that $A / m$ is separably generated over $V / \pi V$. Then $\mathbb{A}_{i}(A)=0$ for $0 \leq i<\operatorname{dim} A \cdot\left(m_{V}=\pi V\right)$.

Proof. Let $P$ be a prime ideal of height $\operatorname{dim} A-i$. If $\pi \in P$, then $[P / \pi]=0$ in $A / \pi A$ by Theorem 4.1 and hence $[P]=0$ in $\mathbb{A}_{i}(A)$. So assume $\pi \notin P$. Choose $a(\neq 0)$ in $P \cap m^{2}$ such that $\{\pi, a\}$ form an $A$-sequence. We then construct $(B, n)$ as in Theorem 1.3 and are done by similar arguments as in the proof of Theorem 4.1 .

\section{ACKNOWLEDGEMENT}

I am thankful to P. Griffith and M. Hochster for their comments and suggestions.

\section{REFERENCES}

[A] M. Artin, Algebraic approximation of structures over complete local rings, I.H.E.S. Sci. Pub. Math. Paris 36 (1969), 23-58. MR 42:3087

[B] P. Berthelot, Altérations de variétes algébriques [d'après A. J. de Jong], Séminaire Bourbake, 48 ème année, $\mathrm{n}^{0}$ 815, 815-01-815-39. MR 98m:14021

[C-E] H. Cartan and S. Eilenberg, Homological Algebra, Princeton University Press, 1956. MR 17:1040e

[C-F] L. Claborn and R. Fossum, Generalizations of the notion of class group, Illinois J. Math. 12 (1968), 228-253. MR 37:200

[D1] S. P. Dutta, A special case of positivity, Proc. Amer. Math. Soc. 103 No. 2 (June 1988). MR 89e:13028

[D2] S. P. Dutta, On Chow groups and intersection multiplicity of modules II, J. Algebra 171 (1995), 370-382. MR 97m:13046

[D-H-M] S. P. Dutta, M. Hochster, J. E. Mclaughlin, Modules of finite projective dimension with negative intersection multiplicities, Invent. Math 79 (1985), 253-291. MR 86h:13023

[F] W. Fulton, Intersection theory, Springer-Verlag, Berlin/Heidelberg/New York/Tokyo (1984). MR 85k:14004 
[G-L] H. Gillet and M. Levine, The relative form of Gersten's conjecture over a discrete valuation ring: The smooth case, J. Pure Appl. Algebra 46 1987, pp. 59-71. MR 88f:18014]

[G-S] H. Gillet and S. Soulé, $K$ theorie et nullite des multipliciités d'intersection, C. R. Acad. Sci., Paris Ser. I 300 (1985), 71-74.

[H1] M. Hochster, Cohen-Macaulay modules, Conference on Commutative Algebra Lecture Notes in Math, Springer, Berlin/Heidelberg/New York 316 (1973), 120-152. MR 49:5006

[H2] M. Hochster, Nonnegativity of Intersection Multiplicities in Ramified Regular Local Rings following Gabber/De Jong/Berthelot, preprint.

[K] K. Kurano, An approach to the characteristic free Dutta Multiplicity, J. Math. Soc. Japan 45 (3) (1993), 369-390. MR 94d:13026

[L] S. Lichtenbaum, On the vanishing of Tor in regular local rings, Illinois J. of Math. 10 (1966), 220-226. MR 32:5688

[Li] H. Lindel, On the Bass-Quillen conjecture concerning projective modules over polynomial rings, Invent. Math. 65 (1981), 319-323. MR 83g:13009

[M] M. P. Malliavin Brameret, Une remarque sur les anneaux locaux réguliers in, 24th Sém. Dubreil-Pisot (Algébre et Théorie des Nombres) No. 13 (1970/71). MR 53:406

[Mat] H. Matsumura, Commutative algebra, Second ed., The Benjamin/Cummings Publish Company (1980). MR 82i:13003

[N] M. Nagata, Local rings, Kriéger, New York (1975). MR 57:301

[Nas] B. Nashier, Efficient generation of ideals in polynomial rings, J. Algebra 85 (1983), 287-302. MR 85f:13010

[P-S] C. Peskine and L. Szpiro, Dimension projective finie et cohomologie locale, Inst. Hautes Etudes Sci. Publ. Math. 42 (1973), 49-119. MR 51:10330

[Q] D. Quillen, Higher algebraic K-theory: I; Algebraic K-theory I-Higher K-theories, Lecture notes in Math. 341, Springer-Verlag, Berlin/Heidelberg/New York/Tokyo. MR 49:2895

[R] P. Roberts, The vanishing of intersection multiplicities of perfect complexes, Bull. Amer. Math. Soc. 13 (1985). MR 87c:13030

[S] J. P. Serre, Algèbre local, multiplicités, Lecture Notes in Math., vol. 11, 3rd ed., Springer-Verlag Berlin/Heidelberg/New York, 1975. MR 34:1352

Department of Mathematics, University of Illinois, 1409 West Green Street, UrBANA, ILLINOIS 61801

E-mail address: dutta@math.uiuc.edu 